\title{
COMUNIDADE DE MACRO-INVERTEBRADOS DO SOLO EM PLANTÍOS FLORESTAIS E SISTEMAS NATURAIS NA AMAZÔNIA PERUANA
}

\author{
Sandra C. TAPIA-CORAL ${ }^{1}$, Flávio LUIZÃO ${ }^{1}$, Beto PASHANASI ${ }^{2}$, Dennis DEL CASTILLO ${ }^{3}$ \\ 1 Instituto Nacional de Pesquisas da Amazônia, Programa de Grande Escala da Biosfera-Atmosfera na Amazônia, \\ INPA/LBA, Av. André Araújo 2936, Campus II, Aleixo, CEP 69060-001, Manaus, AM. Brasil. E-mail: sctcoral@gmail.com \\ 2 Universidad Nacional de la Amazonia Peruana - UNAP, Facultad de Zootecnia, Yurimaguas, Peru \\ 3 Instituto de Investigaciones de la Amazonía Peruana (IIAP), Programa de Investigación en Manejo Integral del Bosque y \\ Servicios Ambientales (PROBOSQUES). Apartado Postal 784, Iquitos, Perú.
}

\section{RESUMO}

Avaliou-se a comunidade dos macro-invertebrados do solo e as propriedades químicas do solo em plantios florestais de Cedrelinga catenaeformis e Simarouba amara comparando-as com uma floresta primária e uma floresta secundária em Jenaro Herrera, na Amazônia peruana. Os macro-invertebrados foram coletados pelo método recomendado pelo Programa de Biologia e Fertilidade de Solos Tropicais (TSBF) e o solo com um trado metálico durante o período de maiores precipitações em 2001. Os valores da densidade (expressados em indivíduos $/ \mathrm{m}^{-2}$ ) dos macro-invertebrados foram maiores nos plantios florestais de Simarouba amara (3702 ind. $\mathrm{m}^{-2}$ ) e de Cedrelinga catenaeformis (2176 ind. $\mathrm{m}^{-2}$ ) do que na floresta primaria e floresta secundaria respectivamente. Assim, destaca-se que os plantios florestais mesmo em forma de monocultivo, no qual a ciclagem de nutrientes ficou restrito aos compostos da própria espécie, apresentaram um efeito favorável na composição dos macro-invertebrados e nas propriedades químicas do solo, mostrando um efeito positivo na recuperação do solo.
\end{abstract}

PALAVRAS-CHAVE: Diversidade de macro-invertebrados, nutrientes do solo, plantios florestais, Cedrelinga catenaeformis, Simarouba amara.

\section{SOIL MACROINVERTEBRATES COMMUNITY IN TREE PLANTATIONS AND MIXED FORESTS IN PERUVIAN AMAZON}

\begin{abstract}
Soil macroinvertebrates community and soil chemical properties were assessed on Cedrelinga catenaeformis and Simarouba amara plantations, and compared to primary and secondary forests in Jenaro Herrera, Peruvian Amazon. Macroinvertebrates was sampled using the TSBF method during the rainy season and surface soil samples were token and analyzed for granulometric composition, acidity, carbon and nutrients. Macroinvertebrates density values where higher on Simarouba amara (3702 ind. $\mathrm{m}^{-2}$ ) and Cedrelinga catenaeformis (2176 ind. $\mathrm{m}^{-2}$ ) plantations than in primary and secondary forests, suggesting favorable effects of forest plantations on soil macroinvertebrates composition and soil chemistry. However, plantation of species both had lower diversity of taxonomic group than primary forest and lower biomass than both primary and secondary forest indicating that tree plantations are still in the process of recovering soil biological characteristics.
\end{abstract}

KEYWORDS: Fauna diversity; soil nutrients; forest plantations; Cedrelinga catenaeformis; Simarouba amara. 


\section{INTRODUÇÃO}

Nas florestais tropicais, a comunidade de macroinvertebrados do solo é muito diversa e compreende milhões de invertebrados que vivem dentro e na superfície do solo ou que passam uma ou mais fases ativas de sua vida no solo. Desempenham um papel chave nos processos que determinam a fertilidade e a estruturação física do solo e regulam a disponibilidade dos nutrientes assimiláveis pelas plantas (Lavelle et al., 1997). Porém, a estrutura e abundância de comunidades de macroinvertebrados do solo são muito sensíveis às diferentes práticas de manejo do solo (Lavelle \& Pashanasi, 1989, Lavelle et al., 1992). As espécies coletadas em distintos ecossistemas podem ter sua atividade diferenciada no solo (Kang et al., 1994). Os organismos das camadas superiores do solo, através de seu papel na decomposição da liteira e na liberação dos nutrientes, são de importância crucial para as condições de crescimento das espécies plantadas e o desenvolvimento e funcionamento dos agroecossistemas (Tian et al.; 1997; Tapia-Coral et al., 1999), principalmente pelas suas interações com a microflora e nutrientes do solo. Uma abundante e diversificada fauna do solo pode ajudar a assegurar uma eficiente reciclagem dos nutrientes (Fittkau \& Klinge, 1973) e um rápido crescimento das plantas (Spain et al., 1992), particularmente em áreas cultivadas de solos inférteis e/ou com baixos insumos (Vohland \& Schroth, 1999; Tapia-Coral, 1999; Tapia-Coral, 2004).

O objetivo do estudo foi analisar o efeito de plantios florestais com respeito à recolonização dos macroinvertebrados e as propriedades químicas do solo, comparando-os com a floresta primária e a floresta secundária de terra firme na Amazônia peruana. Nossa hipótese foi que os plantios florestais, mesmo sendo na forma de monocultivo, poderiam ter um efeito favorável no desenvolvimento de uma abundante e diversa fauna do solo, que poderia ajudar a conservação e o manejo da fertilidade do solo.

\section{MATERIAIS E METODOS}

$\mathrm{O}$ estudo foi realizado no Centro de Investigaciones de Jenaro Herrera (CIJH) $\left(3^{\circ} 39^{\prime} \mathrm{S}, 7^{\circ} 48^{\prime} \mathrm{W}\right)$, do Instituto de Investigaciones de la Amazonía Peruana (IIAP), com uma área de $2567 \mathrm{ha}, 200 \mathrm{~km}$ acima da cidade de Iquitos, na margem direita do Rio Ucayali, na província de Requena, distrito de Jenaro Herrera, Região de Loreto, Peru.

Durante o período de estudo (2001), registrou-se uma precipitação anual de $2674 \mathrm{~mm}$, variando de $410 \mathrm{~mm}$ (março) a $129 \mathrm{~mm}$ (setembro). A temperatura média variou de $20.3{ }^{\circ} \mathrm{C}$ (junho) a 32.5 ${ }^{\circ} \mathrm{C}$ (setembro) (Figura 1).
$\mathrm{O}$ delineamento experimental foi estabelecido em quatro sistemas com três repetições em blocos de 50 x $50 \mathrm{~m}^{2}$, todas em terra firme. Os plantios florestais de Cedrelinga catenaeformis (Ducke) Ducke (C) e Simarouba amara Aublet (S) de 15 e 16 anos de idade respectivamente, estão instalados sobre Ultissolos, em ladeiras de terraços altos, caracterizados por um $\mathrm{pH}$ muito ácido e com uma disponibilidade de nutrientes muito baixa (Claussi et al., 1992). A floresta primária (F) é do tipo densa tropical úmida e, a floresta secundária (SF) abandonada há aproximadamente 16 anos, se estabeleceu sobre cultivos abandonados, apresentando uma vegetação pioneira típica, dominada por Vismia sp. e Cecropia sp.

Foram realizadas três amostras compostas de solo (contendo 10 sub-amostras cada uma, em forma de zigue-zague) na camada de $0-10 \mathrm{~cm}$ de profundidade, com auxílio de um trado metálico. Posteriormente, tratadas e armazenadas para análises granulométricas, de macro e micronutrientes do solo (Tapia-Coral, 2004), no Laboratório Temático de Solos e Plantas do INPA. Todas as análises seguiram o padrão de metodologias de análises físicas e químicas proposta pela Embrapa (1997).

Os macro-invertebrados do solo foram coletados pelo método do Programa de Biologia e Fertilidade de Solos Tropicais (TSBF/IUBS/UNESCO) (Anderson \& Ingram, 1993), coletando-se cinco monolitos de solo de $25 \times 25 \times 30 \mathrm{~cm}$, em todos os sistemas, separados a cada $5 \mathrm{~m}$ ao longo de um transeto em linha reta de $30 \mathrm{~m}$. Os monolitos foram subdivididos em quatro estratos: liteira; camadas do solo de 0-10, 10-20 e 20-30 cm de profundidade. Os macro-invertebrados ( $>2 \mathrm{~mm}$ de diâmetro) foram separados por triagem manual diretamente no campo e armazenados em frascos com álcool a 70\% $+1 \%$ de formol. A identificação foi feita a nível de grandes grupos taxonômicos (Barnes, 1984) e os invertebrados foram contados para estimar sua densidade (ind. $\mathrm{m}^{-2}$ ) e pesados para obter a biomassa fresca em álcool (g.m $\left.{ }^{-2}\right)$, numa balança analítica. As formas imaturas visíveis a olho nu foram separadas, contadas, pesadas e agrupadas como larvas.

Foram realizadas análises de variância (ANOVA) no programa Systat 10.5, previa normalização dos dados para $\log (\mathrm{x}+1)$ (Zar, 1974), com subseqüentes testes de Tuckey ao nível de significância de 1 e 5 \% para verificar diferenças entre os sistemas estudados. Também para cada grupo de variáveis (densidade e biomassa dos macro-invertebrados e as propriedades químicas do solo) foi realizado análises de componentes principais (PCA), associadas com análise discriminante para testar a diferença entre eles. As análises multivariadas foram efetuadas no programa ADE-4 (Thioulouse et al., 1997). 


\section{RESULTADOS}

A granulometria foi variada nos quatro sistemas, sendo que a argila foi significativamente maior na floresta secundária do que nos demais sistemas. $\mathrm{O}$ limo foi significativamente maior no plantio de Cedrelinga catenaeformis e o porcentagem de areia na floresta primária do que nos demais sistemas. $\mathrm{O}$ $\mathrm{pH}$ em $\mathrm{H}_{2} \mathrm{O}$ e $\mathrm{KCl}$ não apresentaram diferenças significativas entre os sistemas. As concentrações de $\mathrm{Al}$ foram maiores na floresta primária e secundária e no plantio de Cedrelinga catenaeformis do que no plantio de Simarouba amara. A acidez foi quase similar em todos os sistemas (Tabela 1).

As concentrações de carbono (C) e nitrogênio $(\mathrm{N})$ no solo foram similares nos plantios florestais e diferiram da floresta primária e secundária. $\mathrm{O}$ fósforo $(\mathrm{P})$ seguiu a mesma tendência que o $\mathrm{C}$ e $\mathrm{N}$, sendo significativamente maior nos plantios florestais que na floresta primária e secundária. As concentrações de potássio $(\mathrm{K})$ foram muito baixas em todos os sistemas, sendo significativamente maior nos plantios florestais que na floresta primária e secundária. O Cálcio $(\mathrm{Ca})$ foi significativamente maior no plantio de Cedrelinga catenaeformis do que nos demais sistemas. O Magnésio $(\mathrm{Mg})$ foi significativamente maior também no plantio de Cedrelinga catenaeformis e floresta secundária do que nos demais sistemas (Tabela 1).

Os dois primeiros fatores explicaram $66.7 \%$ do total da variância, contribuindo com $45.8 \%$ no primeiro fator e $20.8 \%$ no segundo fator. C, N, P, K e o limo foram as variáveis que mais influenciaram no primeiro fator, principalmente nos plantios florestais de Cedrelinga catenaeformis e Simarouba amara. A argila, acidez e o $\mathrm{Mg}$ na floresta secundária e o $\mathrm{pH}$ $\mathrm{H}_{2} \mathrm{O}$ e a areia na floresta primária. No fator $2, \mathrm{o} C \mathrm{Ca}$ foi o nutriente que mais aportou na explicação da variância nos plantios de Cedrelinga catenaeformis Simarouba amara (Figura 2a,b). A análise discriminante do resultado do PCA das propriedades químicas do solo nos diferentes sistemas confirmou a diferença significativa ao nível de $\mathrm{p}<0.005$ (Figura 2b).

Os dois primeiros fatores explicaram $34.4 \%$ do total da variância. O Fator 1 explicou $17.8 \%$ da variância e distingue as comunidades dominadas pelas densidade de cupins (Term) e a densidade e biomassa de formigas (Ant e Antb) nos plantios de Cedrelinga catenaeformis e Simarouba amara de um lado e as densidades de aranhas (Spi) e isópodos (Isp) e a densidade e biomassa de coleópteros (Col e Colb) na floresta primária e secundária ao outro lado (Figura 3a,b). O Fator 2 explicou 16.6\% da variância e separou de um lado as densidades da fauna comedores de liteira (litter-dwelling) como as densidades de hemipteros (Hem), baratas (Blat) e dípteros (Dip) e as densidade e biomassas de minhocas (Erw e Ewmb), e de outro lado a biomassa de diplópodos (Diplb) e isópodos (Ispb) na floresta primária e secundária principalmente (Figura 3a,b). A análise discriminante confirmou a diferença significativa entre os sistemas estudados com uma probabilidade de $\mathrm{p}<0.001$ (Figura $3 b$ ).

Não houve diferença significativa para os valores de densidade entre os sistemas (Tabela 2). Os cupins e formigas foram os grupos predominantes em todos os sistemas estudados, com densidades mais altas nos plantios de Cedrelinga catenaeformis e Simarouba amara. A densidade das minhocas foi significativamente maior entre os sistemas, maior na floresta secundária e primária (106 ind. $\mathrm{m}^{-2}$ ) que nos plantios florestais $(\mathrm{F}=3.89, \mathrm{p}<0.001)$. A densidade dos isópodos e aranhas não apresentaram diferenças significativas entre os sistemas, porém observou-se que ambos grupos foram mais abundantes na floresta primária e no plantio de Cedrelinga catenaeformis. A densidade dos diplópodos também não apresentou diferenças significativas entre os sistemas entretanto, observou-se que foram mais abundantes na floresta secundária e primária do que nos plantios florestais. A densidade dos ortópteros apresentou diferença significativa entre os sistemas $(\mathrm{F}=2.88$, $\mathrm{p}<0.05$ ), sendo maior a densidade na floresta secundária do que nos demais sistemas. Os homópteros também apresentaram diferenças significativas na sua densidade $(\mathrm{F}=3.57, \mathrm{p}<0.05)$ sendo maiores na floresta primária e secundária do que nos plantios florestais (Tabela 2).

A biomassa dos macro-invertebrados do solo apresentou diferenças significativas entre os sistemas $(\mathrm{F}=4.34, \mathrm{p}<0.05)$, sendo maiores na floresta secundária e primária do que nos plantios florestais (Tabela 3). Na floresta primária, a biomassa dos macro-invertebrados foi influenciada pelas minhocas e coleópteros (Tabela 3). A biomassa das minhocas também apresentou diferença significativa entre os sistemas $(\mathrm{F}=5.19, \mathrm{p}<0.05)$, sendo maior na floresta secundária e primária do que nos plantios florestais. As minhocas encontradas foram indivíduos da família Glossoscolecidae, como o Pontoscolex corethrurus na floresta secundária e, exemplares de até $50 \mathrm{~cm}$ de comprimento (minhocoçu) na floresta primária. A biomassa dos diplópodos apresentaram diferenças significativas entre os sistemas $(F=6.78, p<0.001)$, sendo maior na floresta secundária. A biomassa das formigas foi mais alta no plantio de Simarouba, porém sem apresentar diferenças significativas entre os sistemas (Tabela 3). 


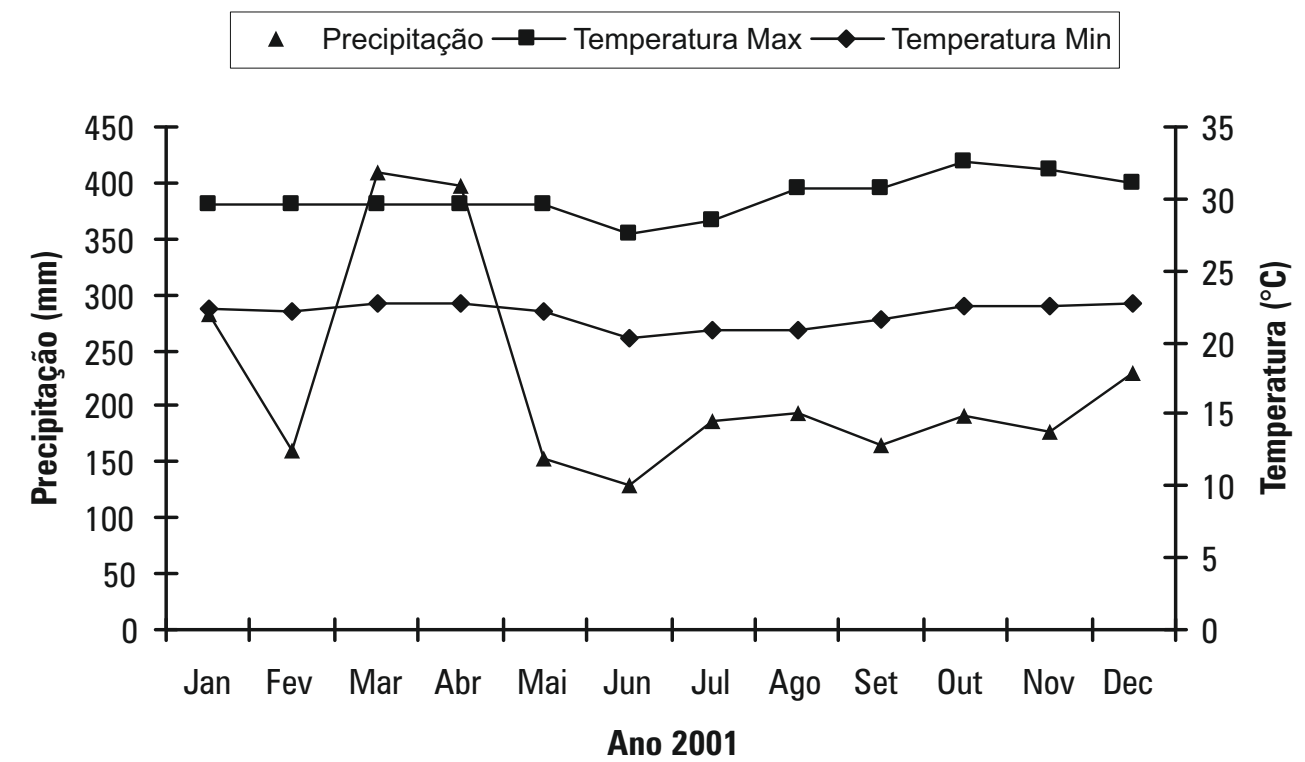

Figura 1. Totais mensais da precipitação pluviométrica $(\mathrm{mm})$ e temperatura máxima e mínima $\left({ }^{\circ} \mathrm{C}\right)$ do ano 2001, registrados na estação meteorológica do ClJH.

Tabela 1. Granulometria, pH, acidez trocável e concentrações de carbono e nutrientes do solo nos plantios florestais de Cedrelinga cataeniformis e Simarouba amara e na Floresta primária e secundária. Os valores são as médias de três amostras compostas e o erro-padrão está entre parênteses. As letras diferentes nas colunas indicam diferenças significativas entre os sistemas ao nível de $5 \%$.

\begin{tabular}{|c|c|c|c|c|c|}
\hline & Cedrelinga & Simarouba & Forest & $\begin{array}{l}\text { Secondary } \\
\text { forest }\end{array}$ & ANOVA \\
\hline Argila (\%) & $30.5(3.1)$ a & $29.8(1.0) \mathrm{a}$ & $23.8(3.5) \mathrm{a}$ & $37.8(2.6) \mathrm{b}$ & $F=52.9 ; p=0.001$ \\
\hline $\operatorname{Limo}(\%)$ & $33.2(2.3) \mathrm{a}$ & $28.5(1.0) \mathrm{b}$ & $25.5(4.4) \mathrm{b}$ & $22.6(0.7) \mathrm{b}$ & $F=37.9 ; p=0.001$ \\
\hline Areia (\%) & $36.3(5.1)$ a & $41.6(1.0) \mathrm{a}$ & $50.6(7.9) \mathrm{b}$ & $39.5(2.0) \mathrm{a}$ & $F=19.3 ; p=0.001$ \\
\hline $\mathrm{pH}\left(\mathrm{H}_{2} \mathrm{O}\right)$ & $3.8(0.1) \mathrm{a}$ & $3.7(0.1) \mathrm{a}$ & $3.9(0.0) \mathrm{a}$ & $3.6(0.1) \mathrm{a}$ & $F=12.0 ; p=0.052 n s$ \\
\hline $\mathrm{pH}(\mathrm{KCl})$ & $3.5(0.1) a$ & $3.5(0.1) a$ & $3.5(0.1) \mathrm{a}$ & $3.6(0.0) \mathrm{a}$ & $F=15.0 ; p=0.055 n s$ \\
\hline $\mathrm{Al}\left(\mathrm{cmol}_{\mathrm{c}} \mathrm{kg}^{-1}\right)$ & $94.1(0.8)$ a & $75.4(0.5) b$ & $95.5(0.6)$ a & $95.3(0.8) \mathrm{a}$ & $F=5.60 ; p=0.05$ \\
\hline Acidez $\left(\mathrm{cmol}_{\mathrm{c}} \mathrm{kg}^{-1}\right)$ & $4.5(0.1) a$ & $4.4(0.2) a$ & $3.5(0.6) b$ & $4.6(0.3) \mathrm{a}$ & $F=22.6 ; p=0.001$ \\
\hline$C\left(\mathrm{~g} \cdot \mathrm{kg}^{-1}\right)$ & $21.1(0.6) \mathrm{a}$ & $22.7(0.3) \mathrm{a}$ & $16.2(2.3) b$ & $18.4(0.8) \mathrm{b}$ & $F=64.6 ; p=0.001$ \\
\hline$N\left(g \cdot \mathrm{kg}^{-1}\right)$ & $1.1(0.0) \mathrm{a}$ & $1.1(0.0) \mathrm{a}$ & $0.8(0.1) b$ & $0.9(0.04) b$ & $F=69.1 ; p=0.001$ \\
\hline$P\left(g \cdot \mathrm{kg}^{-1}\right)$ & $8.48(1.1) \mathrm{a}$ & $7.87(1.18) a$ & $5.84(0.64) b$ & $5.77(0.23) b$ & $F=30.5 ; p=0.001$ \\
\hline $\mathrm{K}\left(\mathrm{cmol}_{\mathrm{c}} \mathrm{kg}^{-1}\right)$ & $0.13(0.02) a$ & $0.11(0.03) a$ & $0.07(0.01) b$ & $0.08(0.01) b$ & $F=29.8 ; p=0.001$ \\
\hline $\mathrm{Ca}\left(\mathrm{cmol}_{\mathrm{c}} \mathrm{kg}^{-1}\right)$ & $0.08(0.01) a$ & $0.06(0.02) \mathrm{b}$ & $0.05(0.01) b$ & $0.06(0.01) b$ & $F=12.6 ; p=0.001$ \\
\hline $\mathrm{Mg}\left(\mathrm{cmol}_{\mathrm{c}} \mathrm{kg}^{-1}\right)$ & $0.07(0.01) a$ & $0.06(0.01) b$ & $0.05(0.01) b$ & $0.07(0.01) \mathrm{a}$ & $F=45.5 ; p=0.001$ \\
\hline
\end{tabular}



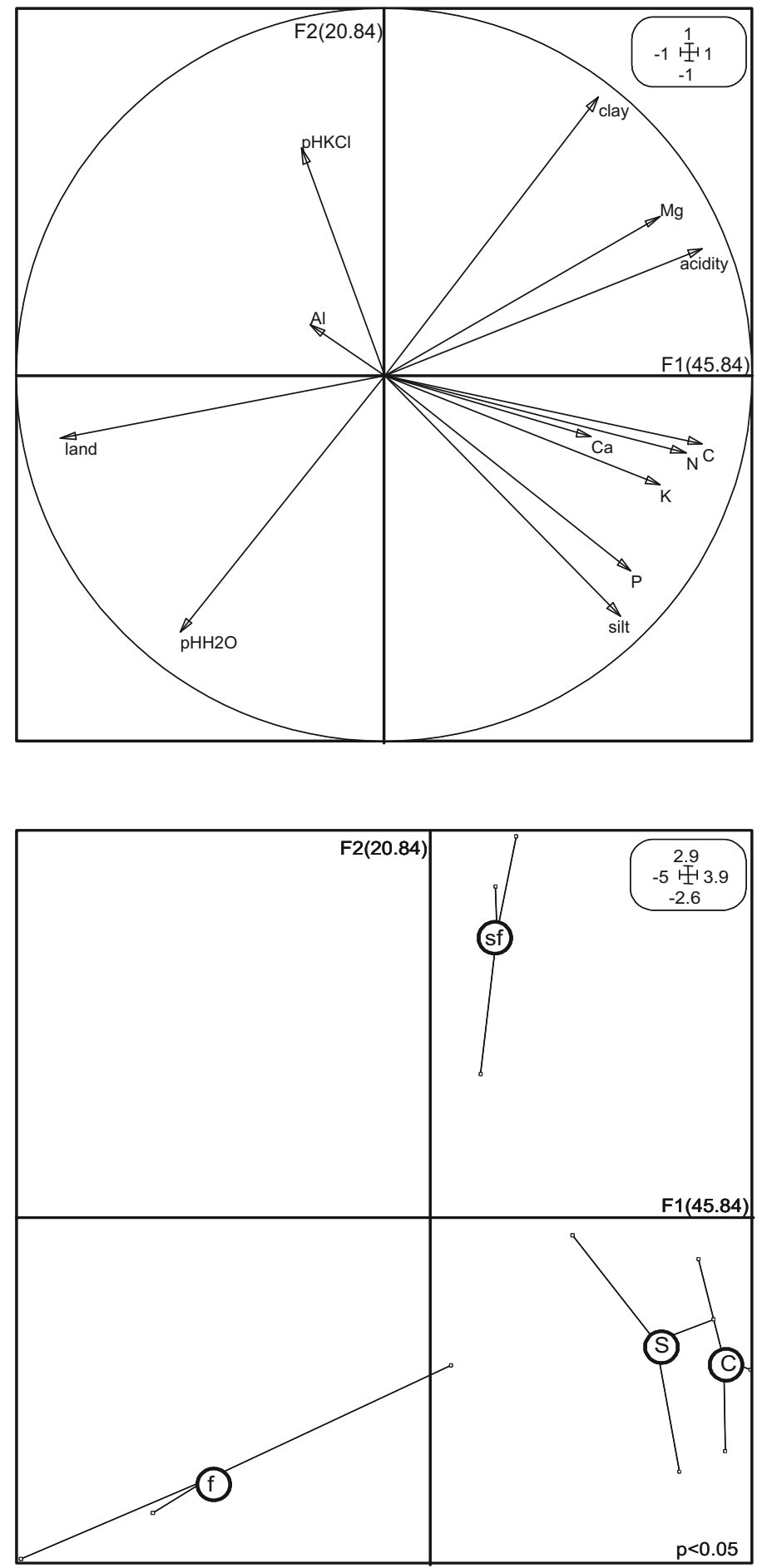

Figura 2a,b. Circulo de correlação das propriedades químicas do solo e distribuição dos sistemas de acordo com os fatores principais 1 e 2. As propriedades químicas do solo estão expressados pela letra inicial de cada um deles e os sistemas correspondem aos plantios florestais de Cedrelinga cataeniformis (C) e Simarouba amara (S) e os sistemas de Floresta primária (F) e Floresta secundária (SF). 

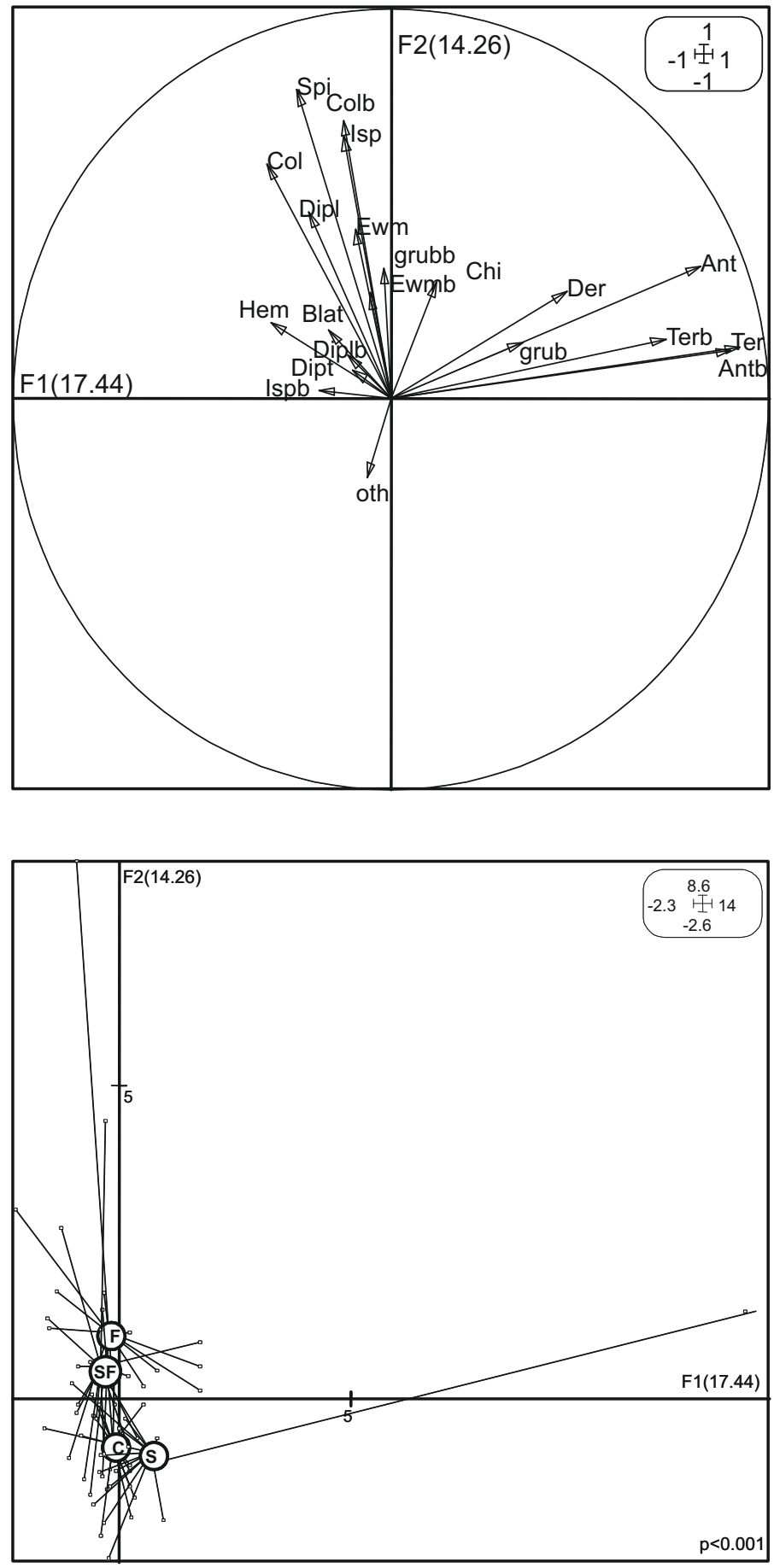

Figure 3a,b. Circulo de correlação da densidade e biomassa dos macro-invertebrados do solo e a distribuição dos sistemas de acordo com os fatores principais 1 e 2. A densidade dos macroinvertebrados está expressada pelas três primeiras letras de cada um deles e a biomassa seguida pela letra $b$ no final. Os sistemas correspondem aos plantios florestais de Cedrelinga cataeniformis (C) e Simarouba amara (S) e os sistemas de Floresta primária (F) e Floresta secundária (SF). 


\begin{tabular}{|c|c|c|c|c|}
\hline Grupos & Cedrelinga & Simarouba & Floresta & $\begin{array}{l}\text { Floresta } \\
\text { Secundaria }\end{array}$ \\
\hline Araneae & $130(38) a$ & $86(28) a$ & $172(66)$ a & $102(28) a$ \\
\hline Blattaria & $3(13)$ a & $60(23)$ a & 45 (18) a & $28(12)$ a \\
\hline Chilopoda & 48 (12) a & 40 (15) a & 42 (14) a & 65 (19) a \\
\hline Coleoptera & $157(33)$ a & $126(28)$ a & $260(53)$ a & $249(51)$ a \\
\hline Dermaptera & $7(4) a$ & $6(5) a$ & $12(9)$ a & $6(4) a$ \\
\hline Diplopoda & $34(10) \mathrm{a}$ & $38(11) a$ & 59 (15) a & 78 (15) a \\
\hline Diptera & $19(12) \mathrm{a}$ & $7(5) a$ & $6(4) a$ & $17(10) \mathrm{a}$ \\
\hline Formicidae & 396 (119) a & $673(348)$ a & $749(250)$ a & $600(159)$ a \\
\hline Homoptera & $10(6)$ a & $7(3) a$ & $29(12) \mathrm{b}$ & $20(11) b$ \\
\hline Isopoda & $90(20)$ a & $53(18)$ a & $143(98)$ a & $49(16)$ a \\
\hline Isoptera & $1065(683)$ a & $2459(2142)$ a & 807 (588) a & 881 (707) a \\
\hline Larvas & $35(14)$ a & 48 (15) a & $30(11)$ a & $37(24)$ a \\
\hline Oligochaeta & $67(41) \mathrm{a}$ & 40 (13) a & $106(43) b$ & $115(44) b$ \\
\hline Orthoptera & $5(4) a$ & $12(9) \mathrm{b}$ & $5(4) a$ & $19(10) b$ \\
\hline Outros & $80(22) \mathrm{a}$ & $47(21) b$ & $16(9) \mathrm{c}$ & $15(11) \mathrm{c}$ \\
\hline Densidade total & 2176 a & $3702 a$ & 2482 a & 2281 a \\
\hline$N^{0}$ Grupos & 20 & 18 & 24 & 19 \\
\hline
\end{tabular}

Tabela 3. Biomassa (g. $\mathrm{m}^{-2}$ ) dos macro-invertebrados do solo nos plantios florestais de Cedrelinga cataeniformis e Simarouba amara e na floresta primária e secundaria. Os valores são as médias de 15 monolitos e 0 erropadrão está entre parênteses. As letras diferentes nas colunas indicam diferenças significativas entre os sistemas ao nível de $5 \%$.

\begin{tabular}{ccccc}
\hline Grupos & Cedrelinga & Simarouba & $\begin{array}{c}\text { Floresta } \\
\text { Primaria }\end{array}$ & $\begin{array}{c}\text { Floresta } \\
\text { Secundaria }\end{array}$ \\
\hline Coleoptera & $0.14(0.08) \mathrm{a}$ & $0.20(0.13) \mathrm{a}$ & $4.08(5.01) \mathrm{b}$ & $0.44(0.27) \mathrm{a}$ \\
Diplopoda & $0.17(0.14) \mathrm{a}$ & $0.06(0.03) \mathrm{a}$ & $0.24(0.18) \mathrm{b}$ & $2.35(1.73) \mathrm{c}$ \\
Formicidae & $0.99(0.52) \mathrm{a}$ & $2.50(2.77) \mathrm{b}$ & $0.83(0.46) \mathrm{a}$ & $0.79(0.33) \mathrm{a}$ \\
Isopoda & $0.03(0.01) \mathrm{a}$ & $0.06(0.05) \mathrm{a}$ & $0.06(0.05) \mathrm{a}$ & $0.02(0.01) \mathrm{a}$ \\
Isoptera & $0.33(0.23) \mathrm{a}$ & $2.30(2.59) \mathrm{b}$ & $2.98(2.96) \mathrm{b}$ & $0.86(0.62) \mathrm{a}$ \\
Larvas & $0.47(0.36) \mathrm{a}$ & $0.56(0.27) \mathrm{a}$ & $0.73(0.56) \mathrm{a}$ & $9.21(8.47) \mathrm{b}$ \\
Oligochaeta & $0.80(0.99) \mathrm{a}$ & $2.20(1.77) \mathrm{b}$ & $4.96(3.92) \mathrm{b}$ & $8.61(6.44) \mathrm{c}$ \\
Outros & $4.08(2.84) \mathrm{a}$ & $1.49(0.62) \mathrm{b}$ & $2.79(1.86) \mathrm{b}$ & $1.94(0.76) \mathrm{b}$ \\
Total & $7.20 \mathrm{a}$ & $9.39 \mathrm{a}$ & $16.7 \mathrm{~b}$ & $24.2 \mathrm{c}$ \\
\hline
\end{tabular}




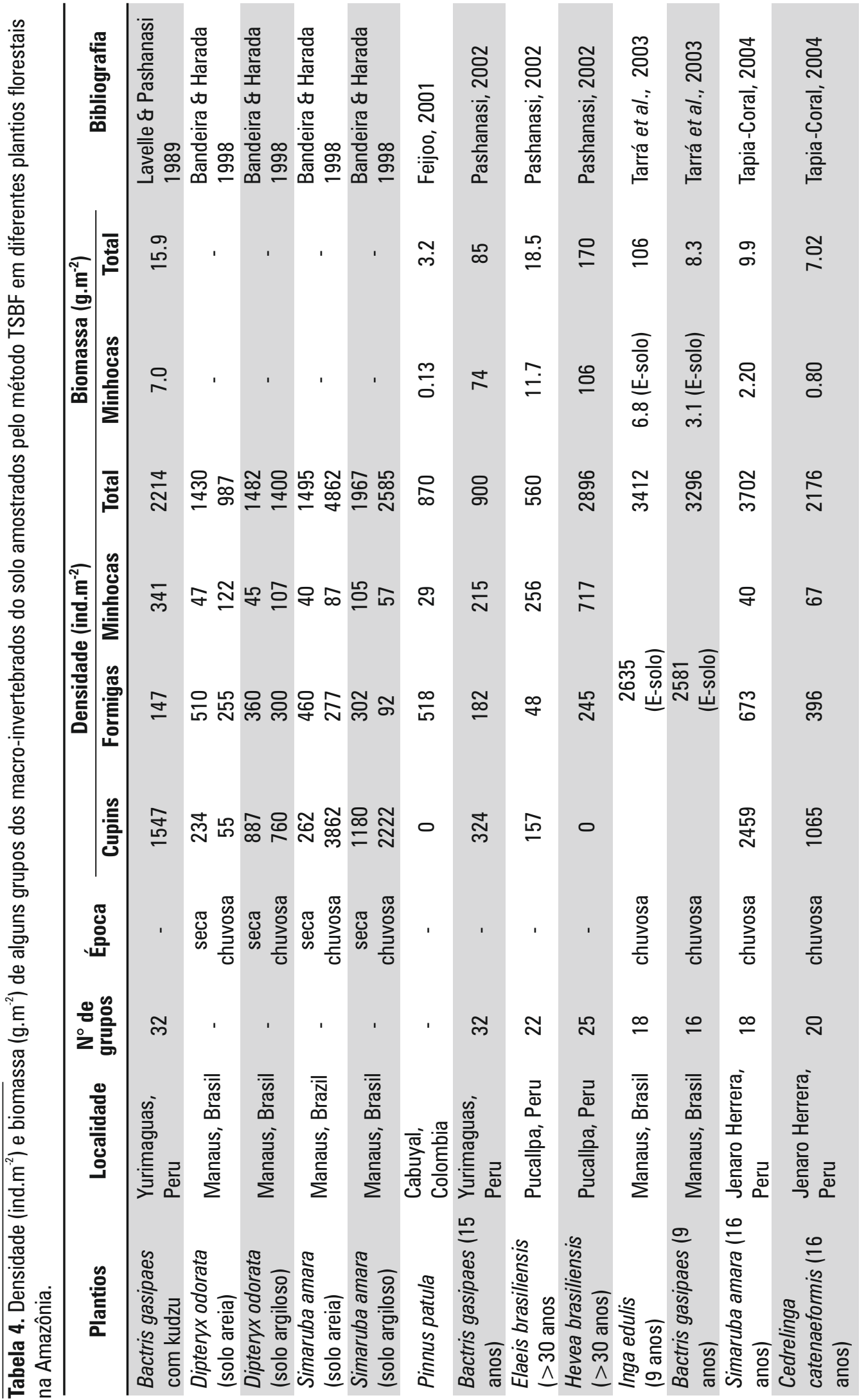




\section{DISCUSÃO}

As concentrações de carbono orgânico no solo foram maiores nos plantios de Cedrelinga catenaeformis e Simarouba amara do que na floresta primária e secundária. As concentrações de carbono nos plantios foram maiores do que sob o solo de Bertholletia excelsa, Theobroma grandiflorum (Schroth et al., 2002) e Carapa guianensis (Morais, 2003) na Amazônia central brasileira. No entanto, Schroth et al. (2002) verificaram a existência de maiores concentrações de carbono orgânico nos primeiros $10 \mathrm{~cm}$ do solo na floresta primária do que numa floresta secundária de 6 anos e um sistema agroflorestal de 7 anos de idade na Amazônia brasileira. As baixas concentrações de nitrogênio encontradas neste estudo podem estar relacionadas às perdas ocasionadas pelo corte e queima que ocorreram nos plantios florestais e na floresta secundária, uma vez que o nitrogênio diminui drasticamente quando a floresta é cortada e queimada (Parton et al., 1989). As concentrações de fósforo no solo também foram relativamente altas principalmente nos plantios florestais de Cedrelinga catenaeformis e Simarouba amara podendo ser produto da rápida decomposição do material vegetal na estação chuvosa (Coleman et al., 1983). As concentrações de potássio, cálcio e magnésio foram muito baixas em todos os sistemas refletindo a pobreza química natural dos solos que, no geral, caracterizam-se pelos baixos conteúdos dos nutrientes. As concentrações de nutrientes do solo deste estudo foram mais baixas do que numa floresta primaria na Amazônia central brasileira (Barros et al., 2003) e em sistemas agroflorestais consorciados de Bactris gasipaes e Theobroma grandiflorum na Amazônia ocidental (Dresch, 2003). No entanto, foram similares as de solos sob plantios de Eucalyptus em diferentes lugares do Brasil (GamaRodrigues \& Barros, 1996). Schroth et al. (2000) afirmaram que seis anos após a instalação de plantios florestais na Amazônia central brasileira precisa-se de uma fertilização urgente de $\mathrm{P}, \mathrm{K}$ e $\mathrm{Mg}$ para o crescimento das árvores, principalmente de Carapa guianensis. Resultados similares também foram reportados por Dunisch et al. (2002), no mesmo local.

Os resultados do PCA das propriedades químicas do solo e da densidade e biomassa da macroinvertebrados foram significativos ao nível de $1 \mathrm{e}$ $5 \%$ respectivamente, onde ambos mostraram um agrupamento dos plantios florestais de Cedrelinga catenaeformis e Simarouba amara, separando-as da floresta primária e secundária. O carbono, nitrogênio, fósforo e potássio foram nutrientes importantes nos plantios florestais de Cedrelinga catenaeformis e Simarouba amara, nas quais também encontrou-se a dominância dos grupos sociais (cupins e formigas) tanto em densidade como em biomassa. Enquanto que a argila, a acidez do solo e o magnésio foram elementos importantes na floresta secundaria. Estudos anteriores também mostraram resultados significativos de PCA, sobretudo quando se compara os macroinvertebrados numa seqüência de diversos sistemas de uso da terra (Lavelle \& Pashanasi, 1989, Decaens et al., 1994; Feijoo et al., 1999; Decaens et al., 2001) e com os parâmetros químicos do solo (Barros et al., 2002).

A maior densidade dos macro-invertebrados foram determinados pelos altos valores de cupins e formigas principalmente nos plantios de Simarouba amara e Cedrelinga catenaeformis. Altas densidades de cupins e formigas também foram encontradas em plantios florestais de Simarouba amara na Amazônia brasileira (Bandeira \& Harada, 1998).

$\mathrm{Na}$ floresta primária, a densidade dos macroinvertebrados foram relativamente baixos e similares a uma floresta primária em Yurimaguas e Pucallpa, na Amazônia peruana (Lavelle \& Pashanasi, 1989; Pashanasi, 2002) e a uma floresta primaria em Manaus (Höfer et al., 2001; Barros et al., 2003) e Rondônia (Barros et al., 2006), na Amazônia brasileira. Porém, uma floresta primária em Marabá na Amazônia oriental foi encontrada uma alta densidade dos macro-invertebrados (Desjardins et al., 2000) (Tabela 4).

A densidade da comunidade dos macroinvertebrados pode variar muito de uma região para outra. Alguns estudos apresentaram valores superiores a este trabalho (Lavelle \& Pashanasi 1989; Bandeira \& Harada 1998; Feijoo, 2001; Pashanasi, 2002) enquanto outros apresentaram valores inferiores (Kato, 1995; Pellens \& Garay, 1999; Pashanasi, 2002) (Tabela 4).

A densidade alta dos cupins no plantio de Simarouba amara poderia atribuir-se ao fato desta madeira branca ser relativamente mole e parecer muito palatável para os cupins (Lee \& Wood, 1971). Os plantios, apesar da idade (15 e 16 anos), ainda não apresentavam suas copas muito fechadas, permitindo o ingresso dos raios solares até o solo (observação pessoal), o que estaria favorecendo a abundância dos cupins. Os cupins são abundantes em solos, onde não haja muita vegetação, desde que haja alimento em abundância (Bandeira \& Harada, 1998).

As formigas foram o segundo grupo mais abundante dos macro-invertebrados, principalmente na floresta primária e secundária seguida pelo plantio de Cedrelinga catenaeformis, o que também concorda com estudos feitos na Amazônia brasileira (Bandeira 
\& Harada, 1998; Barros et al., 2003; Barros et al., 2006). O terceiro grupo mais abundante foram os coleópteros que apresentaram densidades relativamente altas na floresta primária e secundária, porém, em plantios de Pinnus patula e Coffea sp., a densidade e a biomassa dos coleópteros foram relativamente baixos em comparação com o resto dos macro-invertebrados (Feijoo, 2001). As minhocas foram mais freqüentes na floresta secundária e primária, sendo que na floresta secundária os indivíduos pertenciam à espécie Pontoscolex corethrurus, conhecida por encontrarse sempre presente em áreas perturbadas (Lavelle \& Pashanasi, 1989; Feijoo, 2001; Pashanasi, 2002) ou colonizando ambientes altamente degradados (Lavelle et al., 1994; Chauvel et al., 1999). P. corethrurus também foi muito abundante em sistemas perturbados e manejados em Jenaro Herrera (Tapia-Coral et al., 2006).

A biomassa dos macro-invertebrados variou bastante, sendo maiores na floresta secundária e primária seguida dos plantios florestais, porém, menores do que a biomassa encontrada nas florestas primárias e secundárias em Pucallpa (Pashanasi, 2002). As minhocas apresentaram biomassas relativamente baixas na floresta secundária e primária, o que contrasta com um estudo anterior em Manaus, onde uma grande abundância e biomassa de minhocas ocorreram na floresta primária, enquanto que em sistemas de policultivo florestal foram baixas e ocasionais (Römbke et al., 1999). Uma alta biomassa de minhocas com diferentes categorias ecológicas foi registrada em plantações de Bactris gasipaes no México (Fragoso et al., 1997).

A maior diversidade de grupos taxonômicos foi encontrada na floresta primária (24 grupos); o que concorda com o estudo de Lavelle \& Pashanasi (1989) e pode ser devido a maior diversidade de espécies de plantas e de liteira das partes aérea e subterrânea presentes neste ecossistema. A diversidade da fauna geralmente decresce com a intensificação do manejo do solo nos diferentes sistemas de uso da terra, como foi demonstrado na Amazônia peruana (Lavelle \& Pashanasi, 1989; Pashanasi, 2002) num gradiente de diferentes sistemas de usos da terra no Acre e Rondônia, na Amazônia brasileira (Barros et al., 2002; Barros et al., 2006) e com o aumento da degradação do solo na Colômbia (Feijoo et al., 1999, Feijoo, 2001). No entanto, verificou-se que sistemas agroflorestais diversificados apresentam uma maior diversidade e abundância dos macro-invertebrados do que outros tipos de usos da terra, indicando que estes sistemas podem sustentar alta diversidade das comunidades dos macro-invertebrados para otimizar os benefícios dos organismos nestes sistemas (Brown et al., 1999; Tapia-Coral et al., 1999; Barros et al., 2002).
Os resultados mostraram que os plantios florestais, mesmo em forma de monocultivo, no qual a ciclagem de nutrientes ficou restrito aos compostos da própria espécie e da idade que apresentam, mostraram um efeito positivo na recuperação do solo muito melhor que a floresta secundaria (sucessão natural diversificada) de idade similar que foi deixada como pousio. Porém, poderia recomendarse que nos plantios florestais e na floresta secundaria seja considerada a opção de diversificar as áreas com o objetivo de que estes sistemas possam sustentar uma maior diversidade e abundancia dos macroinvertebrados do que outros sistemas de uso do solo.

\section{AGRADECIMENTOS}

Ao Instituto de Investigaciones de la Amazonía Peruana (IIAP) pelo financiamento do estudo. Carlo Magno Shapiama e Hugo Vasquez, do distrito de Jenaro Herrera apoiaram nas coletas de campo. Joel Vasquez e Sandra Ríos colaboraram com a triagem dos macro-invertebrados. O estudo faz parte da tese de doutorado do primeiro autor, que recebeu bolsa do Conselho Nacional de Desenvolvimento Cientifico e Tecnológico-CNPq.

\section{BIBLIOGRAFIA CITADA}

Anderson, J.M.; Ingram, J.S.I. 1993. Tropical Soil Biology and Fertility: a handbook of methods. $2^{\mathrm{a}}$ ed. CAB International. Wallinford, UK. $221 \mathrm{pp}$.

Bandeira, A.G.; Harada, A.Y. 1998. Densidade e distribuição vertical de macro-invertebrados em solos argilosos e arenosos na Amazônia central. Acta Amazônica, 28(2):191-204.

Barnes, D.R. 1984. Zoologia dos invertebrados. $4^{\mathrm{a}}$ Edição, Pensylvania. Roca. 1179pp.

Barros, E.; Pashanasi, B.; Constantino, R.; Lavelle, P. 2002. Effects of land-use system on the soil macrofauna in western Brazilian Amazonia. Biology and Fertility of Soils, (35):338-347.

Barros, E.; Neves, A.; Blanchart E.; Fernandes, E.C.M.; Wandelli, E.; Lavelle, P. 2003. Developement of the soil macrofauna community under silvopastoral and agrosilvicultural systems in Amazonia. Pedobiologia, 47(3):267-274.

Barros, E.; Mathieu, J.; Tapia-Coral, S.C.; Nascimento, A.R.L.; Lavelle, P. 2006. Soil macrofauna communities in Brazilian Amazonia. In: Moreira, F.M.S.; Siqueira, J.O.; Brussaard, L. (Org). Soil biodiversity in Amazonian and other Brazilian ecosystems. 1 ed. Wallingford: CABI Publishing, 1, p. 43-55. 
Brown, G.; Pashanasi, B.; Gilot, C.; Patron, J.C.; Senapati, B.K.; Giri, S.; Barois, I.; Lavelle, P.; Blakemore, A.V.; Spain, A.V.; Boyer, J. 1999. Effects of eartworms on plant production in the tropics. In: Lavelle, P.; Brussard, L.; Hendrix, P. (Eds). Earthworm Managament in Tropical Agroecosystems. CAB International UK. p. 87147.

Chauvel A.; Grimaldi, M.; Barros, E.; Blanchart, E.; Sarrazin, M.; Lavelle, P.; 1999. An Amazonian earthworm compacts more than a bulldozer. Nature, (398):32-33.

Claussi, A.; Marmillod, D. ; Blaser, J. 1992. Descripción silvicultural de las plantaciones forestales de Jenaro Herrera. Instituto de Investigaciones de la Amazonía Peruana (IIAP). Centro de Investigaciones de Jenaro Herrera. Iquitos, Perú. 225pp.

Coleman, D.C.; Reid, C.P.P.; Cole, C.V. 1983. Biological strategies of nutrient cycling in soil systems. Advance Ecology Research, (13):1-55.

Decaens, T.; Lavelle, P.; Jiménez, J.J.; Escobar, G.; Rippstein, G. 1994. Impact of land management on soil macrofauna in the Oriental Llanos of Colombia. European Journal Soil Biology, 30(4):157-168.

Decaens, T.; Jiménez, J.J.; Rangel, A.F.; Cepeda, A.; Moreno, A.G.; Lavelle, P. 2001. La macrofauna del suelo en la sabana bien drenada de los llanos orientales. In: Rippstein, G., Escobar, G., Motta, F. (Eds). Agroecologia y Biodiversidad de las Sabanas en los Llanos Orientales de Colombia. CIAT. p. 111-137.

Desjardins, T.; Lavelle, P.; Barros, E.; Brossard, M.; Chapuis-Lardy, L.; Chauvel, A.; Grimaldi, M.; Guimarães, F.; Martins, P.; Mitja, D.; Muller, M.; Sarrazin, M.; Tavares Filho, J.; Topall, O. 2000. Dégradadion des pâturages amazoniens. Étude et Gestion des Sols, (7):353-378.

Dresch, P. 2003. Influência da Pueraria phaseloides sobre a transformação microbiana do nitrogênio do solo nos sistemas agroflorestais do projeto RECA em Nova Califórnia - RO. Dissertação de mestrado, INPA/UFAM. 53pp.

Dunisch, O.; Erbreich, M.; Eilers, T. 2002. Nutrient fluxes and growth of $C$. guianensis Aubl. in two plantation systems in the central Amazon. Forest Ecology and Management, (166):55-68.

Embrapa. Centro Nacional de Pesquisa de Solos. 1997. Manual de métodos de análise de solo. $2^{\mathrm{a}}$ ed. Rio de Janeiro, RJ. (EMBRAPA CNPS. Documentos; 1). 212pp.

Feijoo, A.M. 2001. Impacto del uso de la tierra en áreas de laderas sobre comunidades de macrofauna del suelo (Caldono, Cauca, Colombia). Tesis de Doctorado. Universidad Nacional de Colombia. 196pp.
Feijoo, A.M.; Bronson Knapp, E.; Lavelle, P.; Moreno, A.G. 1999. Quantifying soil macrofauna in a Colombian watershed. Pedobiologia, (43):513-517.

Fittkau, E.J.; Klinge, H. 1973. On biomass and trophic structure of the Central Amazonian rain forest. Biotropica, 5(1):2-14.

Fragoso, C.; Brown, G.G.; Patrón, J.C.; Blanchart, E.; Lavelle, P.; Pashanasi, B.; Senapati, B.; Kumar, T. 1997. Agricultural intensification, soil biodiversity and agrosystem function in the tropics: role of earthworm. Applied Soil Ecology, (6):17-35.

Gama-Rodigues, A.C., Barros, N.F. 1996. Biomassa e nutrientes da serrapilheira e do solo sob plantios puros e misto de espécies florestais nativas do sudeste da Bahia, Brasil. Resumo do XXVI Congresso Brasileiro de Ciência do Solo. CD ROM, artigo 04-185 (4 pág) Software Gráfico Ltda. Campinas, SP.

Höfer, H.; Hanagarth, W.; Garcia, M.; Martius, C.; Franklin, E.; Rombke, J.; Beck, L. 2001. Structure and function of soil fauna communities in Amazonian anthropogenic and natural ecosystems. European Journal Soil Biology, (37):1-7.

Kang, B.T.; Akinnifesi, K.; Pleysier, J.L. 1994. Effect of agroforestry woody species on earthworm activity and physiochemical properties of worm casts. Biology Fertility and Soils, (18):193-199.

Kato, A.K. 1995. Dinâmica da Entrada de Nutrientes via Liteira em Plantios de Castanheira-do-Brasil (Bertholletia excelsa H.B.K.) em Ecossistemas de Pastagens Degradadas e de Floresta Primária. Tese de Doutorado. INPA/UFAM, Manaus-AM. 180pp.

Lavelle, P.; Pashanasi, B. 1989. Soil macrofauna and land management in Peruvian Amazonia (Yurimaguas, Loreto). Pedobiologia, 33, 283291.

Lavelle, P.; Spain, A.V.; Blanchart, E.; Martin, A.; Martin, S. 1992. The impact of soil fauna on the properties of soils in the humid tropics. In: Myths and Science of Soils of the Tropics. SSSA Special Publication. Madison Wisconsin, p. 157-185.

Lavelle, P.; Dangerfield, M.; Fragoso, C. 1994. The relationship between soil macrofauna and tropical soil fertility. In: Swift, M.J., Woomer, P. (Eds). Tropical Soil Biology and Fertility. Jhon Wiley Sayce. New York. p. 137-169.

Lavelle, P.; Bignell, D.; Lepage, M.; Wolters, V.; Roger, P.; Ineson, P.; Heal, O.W.; Dhillion, S. 1997. Soil function in a changing world: the role of invertebrate ecosystem engineers. European Journal of Soil Biology, (33): 159-193. 
Lee, K.E.; Wood, T.G. 1971. Physical and chemical effects on soils of some Australian termites, and their pedological significance. Pedobiologia, (11): 376-409.

Morais, R.R. 2003. Ecofisiologia de espécies arbóreas crescidas sob condições de plantios na Amazônia central. Tese de Doutorado, INPA/UFAM. 158pp.

Parton, W.J.D.; Sandford Jr., R.L.; Sanchez, P.A.; Stewart, J.W.B.; 1989. Modeling soil organic matter dynamics in tropical soils. In: Coleman, D.C., Oades, J.M.; Uehara, G. (Eds). Dynamics of Soil Organic Matter In Tropical Ecosystems, University of Hawaii Press, Honolulu, Hawaii, p. 153-171.

Pashanasi, B. 2002. Estudio cuantitativo de la macrofauna del suelo en diferentes sistemas de uso de la tierra en la Amazonía Peruana. Folia Amazonica, 12(1-2):75-97.

Pellens, R.; Garay, I. 1999. Edaphic macroarthropod communities in fast-growing plantations of Eucalyptus grandis Hill ex Maid (Myrtaceae) and Acacia mangium Wild (Leguminosae) in Brazil. European Journal of Soil Biology, 35(2):77-89.

Rombke, J.; Meller, M.; Garcia, M. 1999. Earthworm densities in central Amazonian primary and secondary forest and a polyculture forestry plantation. Pedobiologia., (43):518522.

Schroth, G.; Seixas, R.; da Silva, L.F.; Texeira, W.G.; Zech, W. 2000. Nutrient concentrations and acidity in ferralitic soil under perennial cropping, fallow and primary Forest in central Amazoniana. European Journal of Soil Sciences, (51):219-231.

Schroth, G.; D'Angelo, S.A.; Teixeira, W.G.; Haag, D.; Lieberei, R. 2002. Conversion of secondary forest into agroforestry and monoculture plantations in Amazonia: consequences for biomass, litter and soil carbon stocks after 7 years. Forest Ecology and Management, (163):131-150.

Spain, A.V.; Lavelle, P.; Mariotti, A. 1992. Stimulation of plant growth by tropical earthworms. Soil Biology and Biochemistry, (24):1629-1633.

Tapia-Coral, S.C. 2004. Macro-invertebrados do solo e estoques de carbono e nutrientes em diferentes tipos de vegetação de terra firme na Amazônia peruana. Teses de Doutorado. INPA/UFAM. 134pp.

Tapia-Coral, S.C.; Luizão, F.; Wandelli, E. 1999. Macrofauna da liteira em sistemas agroflorestais sobre pastagens abandonadas na Amazônia central. Acta Amazônica., 29(3):477495.

Tapia-Coral, S.C.; Luizão, F.J.; Barros, E.; Pashanasi, B.; Del Castillo, D. 2006. Effect of Pontoscolex corethrurus Muller, 1857 (Oligochaeta: Glossoscolecidae) Inoculation on Litter Weight Loss and Soil Nitrogen in Mesocosms in the Peruvian Amazon. Caribbean Journal of Sciences, 42(3):410-418.

Tarrá, I.L.C.; Luizão, F.J.; Wandelli, E.; Teixeira, W.; Morais, W.J.; Fernandes, E.C.M.; Villalobos, B.K; Varón, N.P. 2011. Grupos funcionais da macrofauna e macroporos do solo em sistemas agroflorestais na Amazônia central. Prospect, 10(1):6-17.

Thioulouse, J. ; Chessel, D. ; Dolédec, S. ; Olivier, J.M. 1997. ADE4 multivariate analysis and graphical display software. Statistical Computation, (7):2-15.

Tian, G.; Brussaard, L.; Kang, B.T.; Swift, M.J. 1997. Soil Fauna-mediated decomposition of plant residues under constrained enviromental and residue quality conditions. In: Cadish, G., Giller, K.E. (Eds). Driven by Nature: Plant Litter Quality and Decomposition. CAB International, UK, p. 125-134.

Volhland, K.; Schroth, G. 1999. Distribution patterns of the litter macrofauna in agroforestry and monoculture plantations in Central Amazonia as affected by plant species and management. Applied Soil Ecology, (13):57-68.

Zar, J.H. 1974. Bioestatistical Analysis. PrenticeHall, Inc., Englewood Cliffs, N. J. 718pp. 